\title{
HEMODYNAMIC ALTERATIONS IN BRONCHIECTASIS: A BASE FOR A NEW SUBCLASSIFICATION OF THE DISEASE
}

M. Ashour, FRCS
On the basis of the morphologic and hemodynamic features in 17 patients with bilateral bronchiectasis, a new subclassification is proposed. Accordingly, two types of bronchiectasis were recognized: perfused and nonperfused. Whereas perfused bronchiectasis has intact pulmonary artery flow and cylindrical bronchiectatic changes, the nonperfused type involves an absent pulmonary artery flow, retrograde filling of the pulmonary artery through the systemic circulation, and cystic bronchiectatic changes. A policy of unilateral resection of nonperfused bronchiectasis and preservation of the perfused type was adopted in 17 patients with bilateral bronchiectasis during an 8-year period. There were 9 women and 8 men with an average age of $28.6 \pm 7$ years (range 18 to 48 years). Fifteen patients had mixed bronchiectasis (perfused type on one side and nonperfused on the other side) and two had localized bilateral nonperfused type. The average duration of follow-up was $38.3 \pm 24.9$ months (range 13 to 111 months). In the 15 patients with mixed bronchiectasis, excellent $(N=8)$ or $\operatorname{good}(N=7)$ results were achieved in all cases. On the other hand, the two patients with bilateral nonperfused bronchiectasis did not benefit from unilateral resection. This outcome implies that with perfused bronchiectasis the deranged function is likely to resolve with time. In the face of the general criticism of the traditional morphologic classification system, the proposed functional classification not only reflects the degree of severity of the disease process, but also predicts whether the involved lung will have a measure of respiratory function with regard to gas exchange. Thus the question of which side to resect and which to preserve is defined more precisely. (J Thorac Cardiovasc Surg 1996;112:328-34)
B ronchiectasis or dilation of the bronchi was first described by Laenec in $1918 .{ }^{1}$ Since then classifications of varying complexities have been proposed. On the basis of examination of morbid specimens, Roles and $\operatorname{Tod}^{2}$ described five types of bronchiectasis: tubular, early fusiform, late fusiform, fusosaccular, and saccular. Later, Reid ${ }^{3}$ described three types of bronchiectasis taking into consideration the findings on bronchography.

The word bronchiectasis as currently used is a

From the Division of Thoracic Surgery, Department of Surgery, King Khalid University Hospital, Riyadh, Saudi Arabia.

Received for publication June 2, 1995; accepted for publication Oct. 2, 1995.

Address for reprints: M. Ashour, FRCS, Associate Professor of Thoracic Surgery, King Khalid University Hospital, P. O. Box 7805, Riyadh 11472, Saudi Arabia.

Copyright 01996 by Mosby-Year Book, Inc.

$0022-5223 / 96 \$ 5.00+0 \quad \mathbf{1 2 / 1 / 6 9 6 6 9}$ descriptive term that fails to indicate which type of the disease may have a measure of respiratory function with regard to gas exchange. Moreover, hemodynamic alterations specific to each type of bronchiectasis, to my knowledge, have not been reported before. To investigate these aspects of the disease, a prospective study of 17 patients with bilateral bronchiectasis was undertaken. On the basis of these hemodynamic alterations, a new subclassification is proposed. Its impact on the surgical treatment of patients with bronchiectasis is also discussed.

\section{Patients and methods}

Seventeen patients with bilateral bronchiectasis were studied prospectively, before operation, during a period of 8 years (1987 to 1993). Computed tomography (CT) of the chest and complete bilateral bronchograms were done in all patients to identify the morphologic features of the disease. To determine the various hemodynamic alterations in these patients, ventilation/perfusion (V/Q) lung 

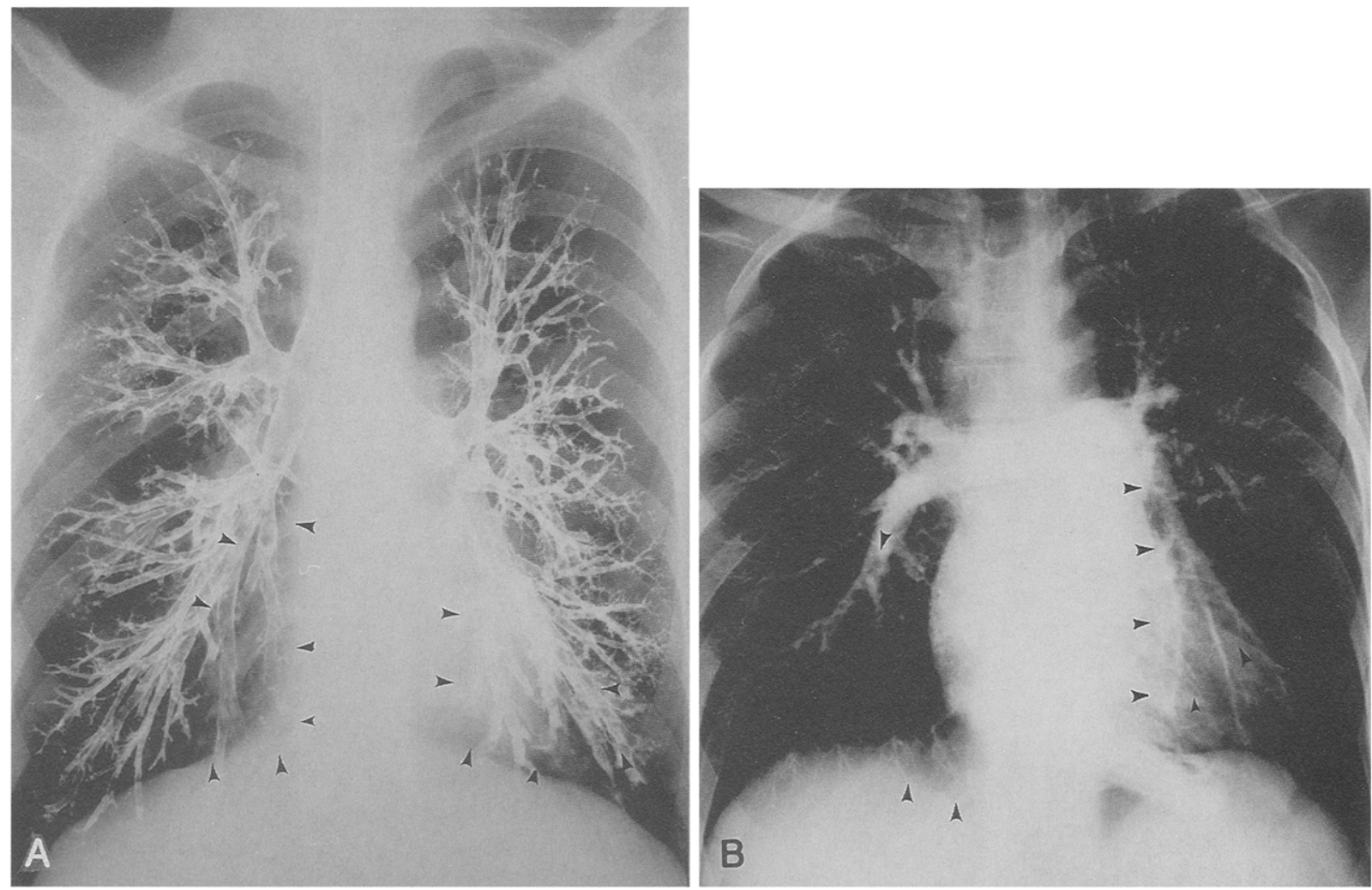

Fig. 1. A, Bronchogram showing cylindrical bronchiectasis of left lower lobe (small arrows) and right medial basal segment. B, Pulmonary angiogram of same patient showing intact perfusion of left lower lobe (perfused bronchiectasis) and of right medial basal segment (both marked by small arrows).

Table I. Operations

\begin{tabular}{lccc}
\hline Type of operation & $\begin{array}{c}\text { Right } \\
\text { side }\end{array}$ & $\begin{array}{c}\text { Left } \\
\text { side }\end{array}$ & $\begin{array}{c}\text { Total } \\
\text { No. }\end{array}$ \\
\hline Lobectomy & 3 & 8 & 11 \\
Lingulectomy and & - & 4 & 4 \\
basal segmentectomy & & & \\
$\begin{array}{c}\text { with preservation of } \\
\text { apical segment }\end{array}$ & & & \\
Pneumonectomy & - & 2 & 2 \\
\hline
\end{tabular}

scan, pulmonary angiography, and thoracic aortography were done. Bronchoscopy was done routinely to rule out endobronchial lesions as a cause for the disease. Cardiorespiratory assessment included blood gas analysis, full pulmonary function tests, and electrocardiograms. Sputum was cultured routinely for pyogenic organisms and tubercular bacilli.

Indications for operation included production of large amounts of sputum and recurrent hemoptysis. After operation, all patients received intensive chest physiotherapy and the appropriate antibiotics. The operative procedures involved unilateral lung resection of the more severely affected side only. This was identified as the side having
Table II. Patients

\begin{tabular}{lccc}
\hline Age $(y r)$ & Men & Women & Total \\
\hline Up to 20 & 2 & 0 & 2 \\
21-30 & 2 & 7 & 9 \\
$31-40$ & 3 & 2 & 5 \\
$41-50$ & 1 & 0 & 1 \\
\hline
\end{tabular}

cystic bronchiectatic changes and being nonperfused according to $\mathrm{V} / \mathrm{Q}$ lung scan and pulmonary angiography. The various operations done on the basis of this selection criterion are shown in Table I. Resected specimens were sent for histologic examinations.

The outcome of operation was evaluated according to the following criteria: excellent, no sputum production since the operation; good, marked reduction in sputum production; and poor, no change in sputum production.

\section{Results}

There were eight men and nine women with an average age of $28.6 \pm 7$ years (range, 18 to 48 years) (Table II). All patients had chronic productive 

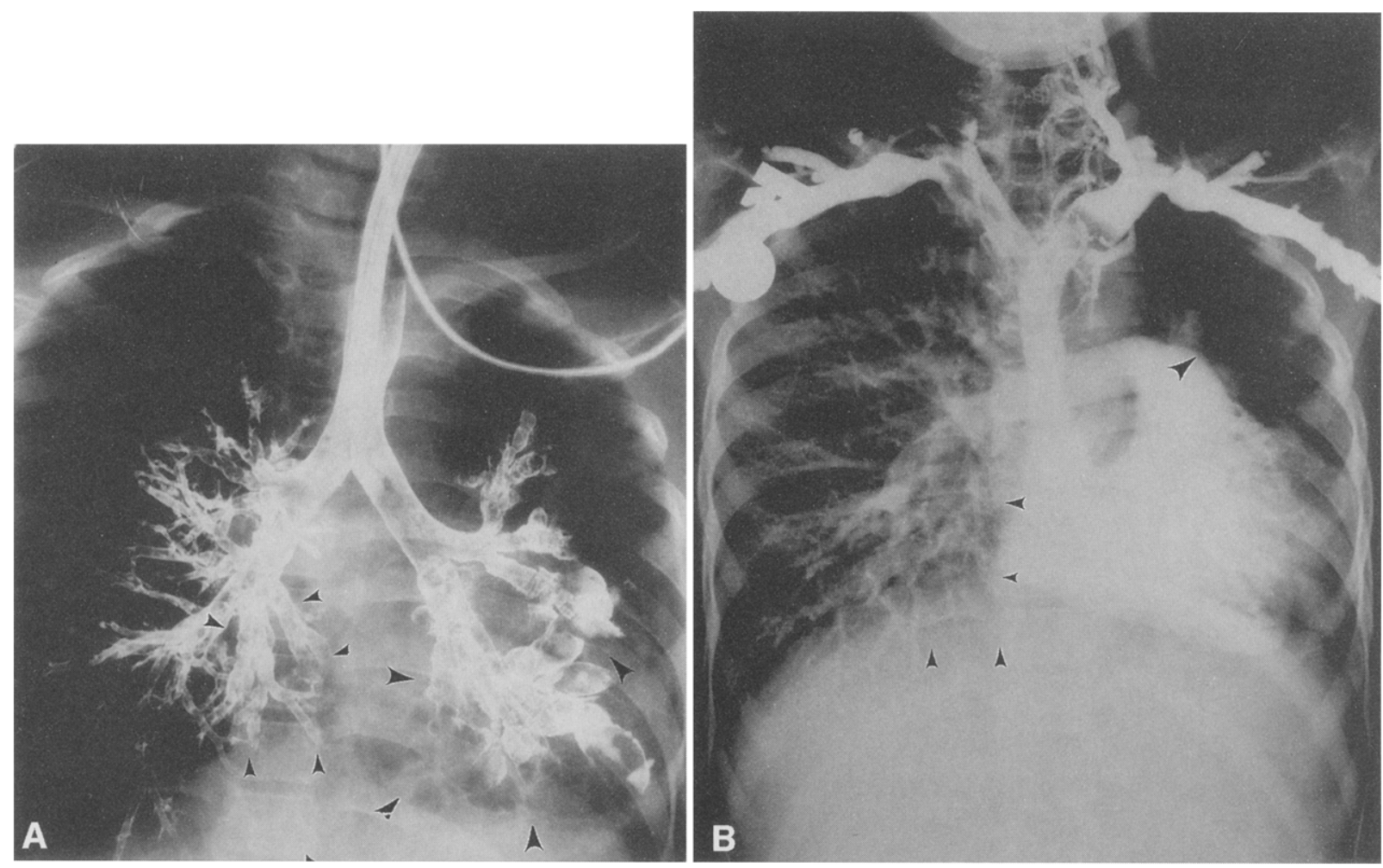

Fig. 2. A, Bronchogram showing cystic bronchiectasis of left lung (large arrows) and cylindrical bronchiectasis of right medial basal segment (small arrows). B, Pulmonary angiogram of same patient showing absent pulmonary artery flow to left lung (nonperfused bronchiectasis) and intact perfusion of right medial basal segment (perfused bronchiectasis), marked by large and small arrows, respectively.

cough for an average period of $12.6 \pm 6.1$ years (range, 2 to 23 years). Ten (58.8\%) of $17 \mathrm{had}$ hemoptysis as well. Severity of hemoptysis ranged from frequent blood-tinged sputum to massive hemoptysis up to $300 \mathrm{ml}$ of fluid. Average duration of hemoptysis was $1.9 \pm 4.5$ years (range, 6 months to 19 years). Sputum culture results were available for 16 patients. The culture grew normal flora in 10 $(58.8 \%)$, Haemophilus influenzae in $4(23.5 \%)$, and Pseudomonas aeruginosa in $2(11.8 \%)$. Sputum was negative for acid fast bacilli in all patients.

On the basis of chest CT and bronchogram, two types of bronchiectatic changes were recognized: cylindrical and cystic. Cylindrical changes were seen in 15 lungs, 13 on the right side and 2 on the left. Cystic changes were found in 19 lungs, 15 on the left side and 4 on the right. Pulmonary angiography showed absent pulmonary artery flow in 19 lungs and intact flow in 15. These findings were matched by V/Q lung scan in all patients. When the morphologic features of bronchiectasis was correlated with the angiographic and $V / Q$ lung scan findings, all lungs with cylindrical bronchiectatic changes were found to be perfused ( $N=15$ lungs) (Fig. 1, $A$ and $B)$, whereas those with cystic changes were nonperfused ( $N=19$ lungs) (Fig. 2, $A$ and $B$ ). Thus in 15 patients bronchiectasis was perfused on one side and nonperfused on the other side (mixed bronchiectasis) (Fig. 3, $A$ and $B$ ). The other two patients had localized bilateral nonperfused bronchiectasis. Thoracic aortography revealed retrograde filling of the pulmonary artery (Fig. $4, A$ and $B$ ) and dilation of bronchial and intercostal arteries in patients with nonperfused bronchiectasis only (Fig. 5, $A, B$, and $C)$. Bronchoscopy did not reveal any endobronchial pathologic conditions in any patient. Intraoperatively, obliteration of pleural spaces with vascular adhesions, as well as lung adherence to chest wall, pericardium, and diaphragm, were common findings. There was no mortality in this series. Histologic examination of resected lungs (nonperfused bronchiectatic lungs) showed extensive cavitations beyond which lay compressed, fibrosed, and chronically inflamed alveolar tissues. Pulmonary capillary 

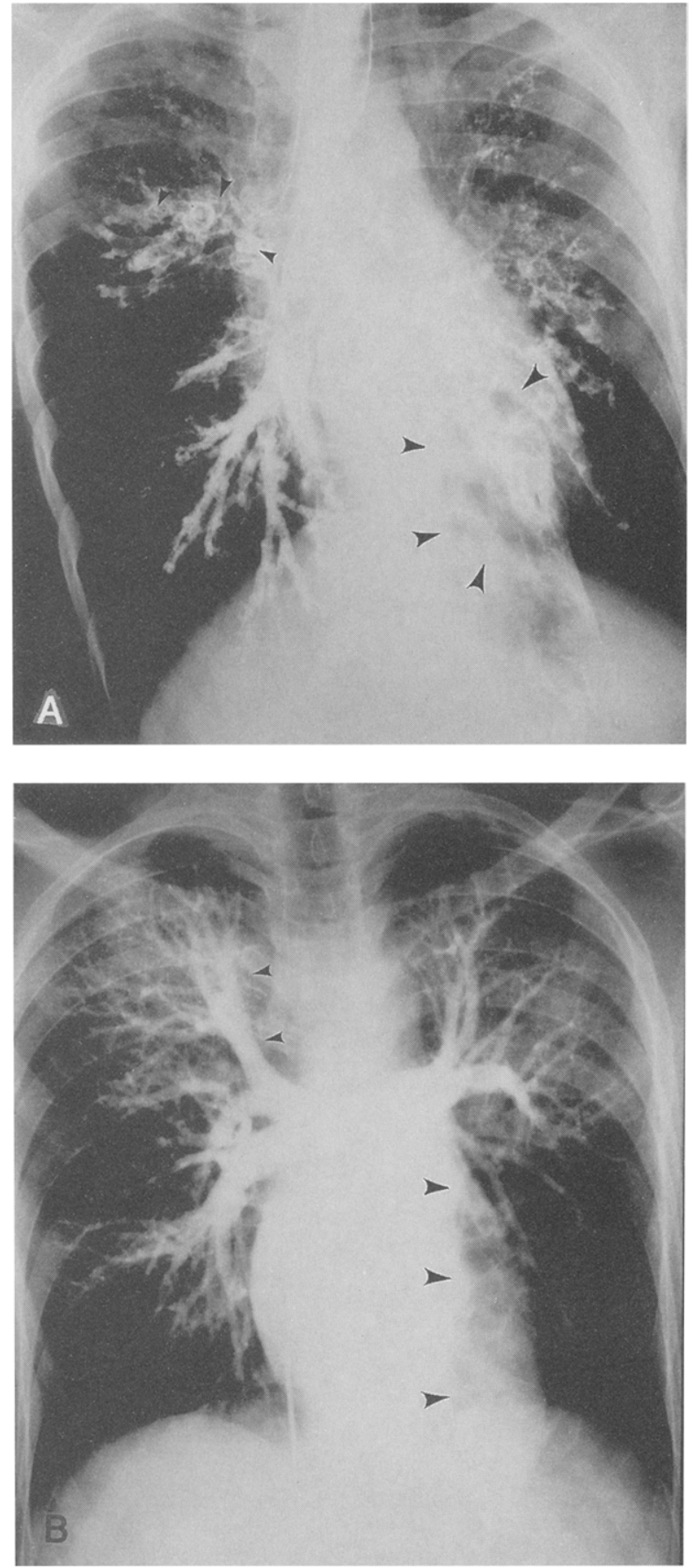

Fig. 3. A, Bronchogram showing cystic and cylindrical bronchiectasis of both left lower lobe (large arrows) and right upper lobe (small arrows). B, Example of mixed bronchiectasis: pulmonary angiogram of same patient showing no flow to left lower lobe (nonperfused bronchiectasis) and intact flow to right upper lobe (perfused bronchiectasis), marked by large and small arrows, respectively.
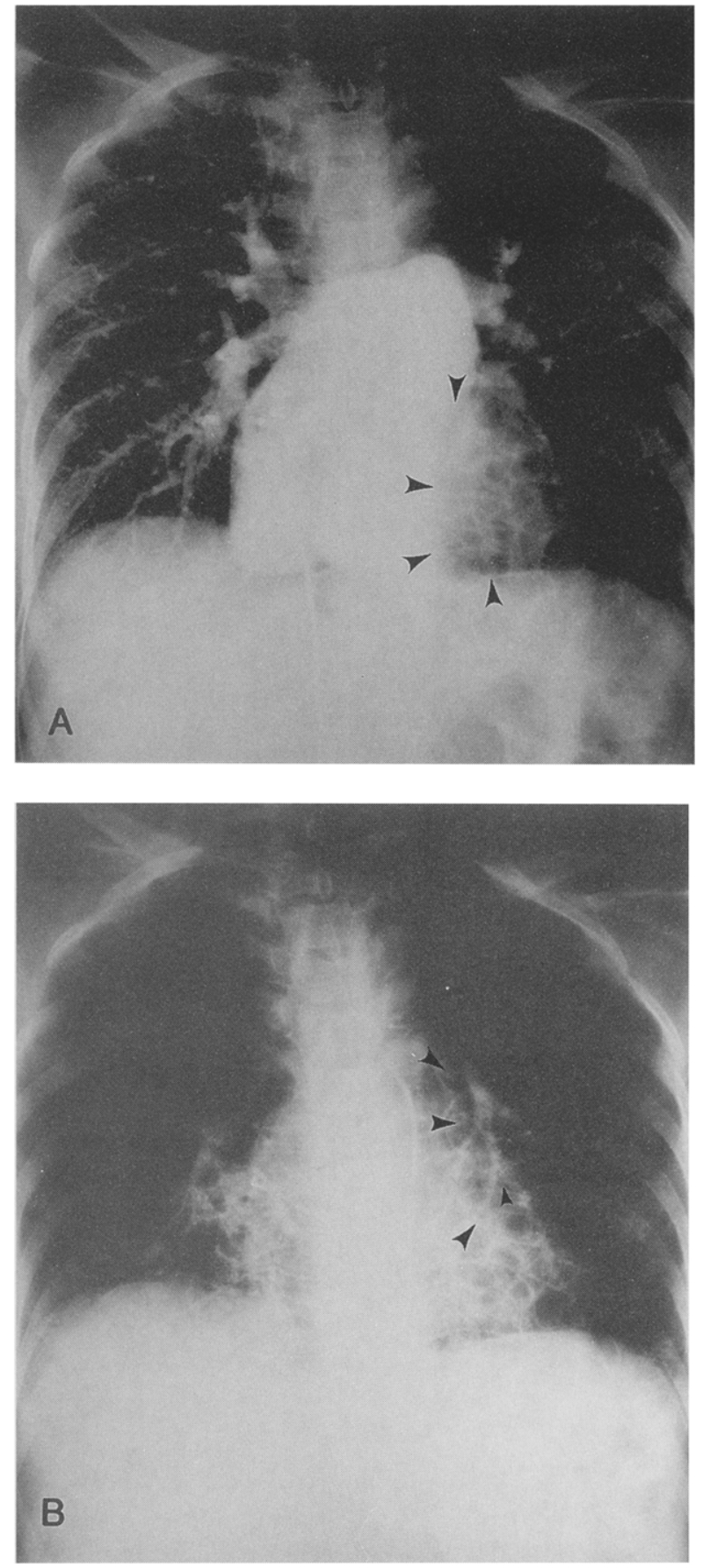

Fig. 4. A, Pulmonary angiogram showing absent pulmonary artery flow to left lower lobe (small arrows) that is involved with cystic bronchiectasis (large arrow). B, Thoracic aortography of same patient showing retrograde filling of pulmonary artery of left lower lobe (small arrows) through systemic circulation. 

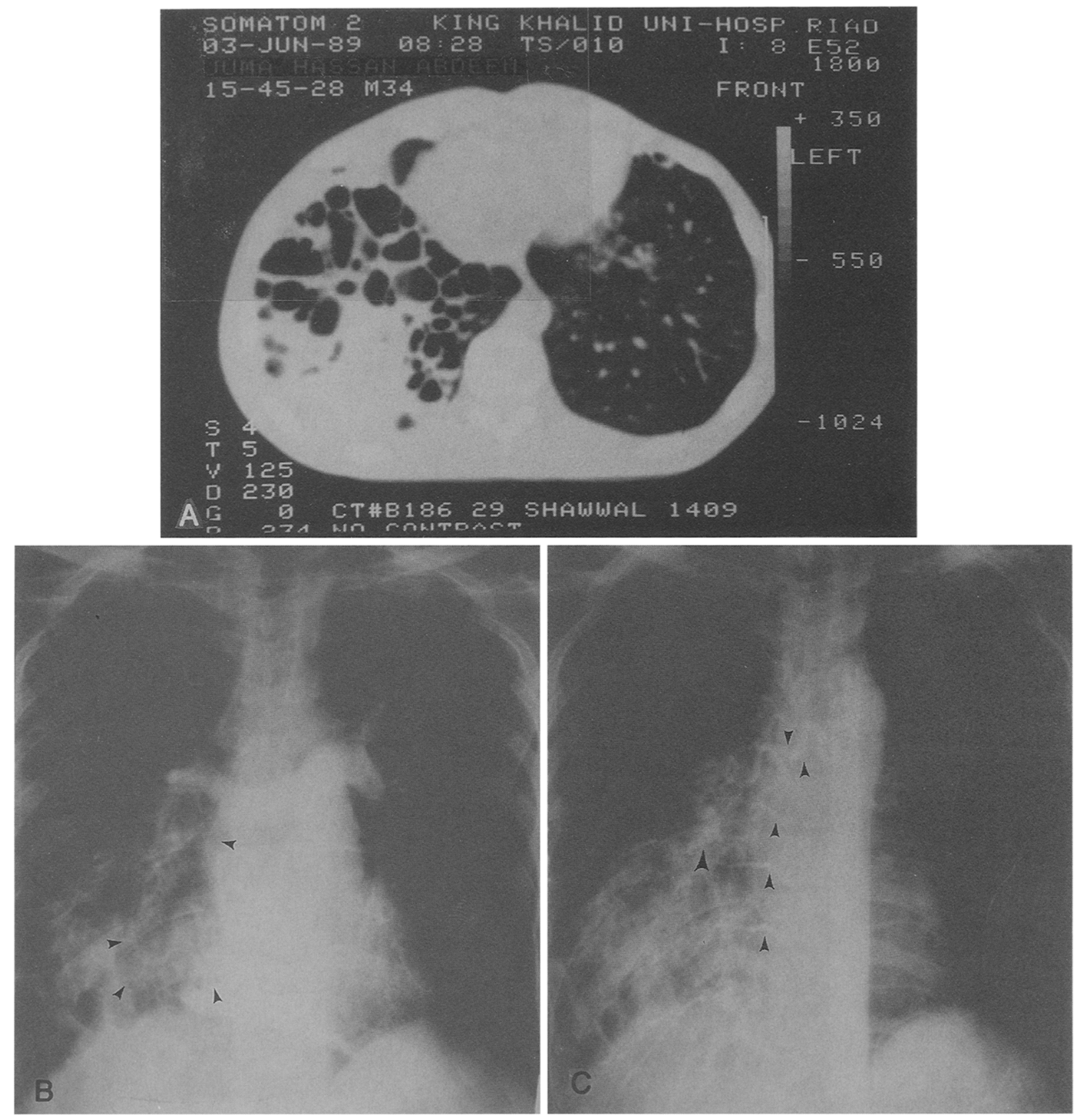

Fig. 5. A, CT of chest showing cystic bronchiectasis of right lower lobe. B, Pulmonary angiogram of same patient showing nonperfused bronchiectasis of right lower lobe (small arrows). C, Thoracic aortography of same patient showing retrograde filling of right lower lobe pulmonary artery (large arrow), with more dilated bronchial and intercostal arteries of right hemithorax (small arrows) than of left hemithorax.

bed was not seen. Dilation and proliferation of bronchial arteries and bronchopulmonary shunt formations were seen in most specimens.

Average duration of follow-up was $38.3 \pm 24.9$ months (range, 13 to 111 months). In 15 patients with mixed bronchiectasis, results were excellent in 8 patients and good in 7 . Seven of 8 patients with hemoptysis were free of symptoms during the period of follow-up. On the other hand, the two patients with localized bilateral nonperfused bronchiectasis did not benefit from unilateral resection and continued to have hemoptysis. 

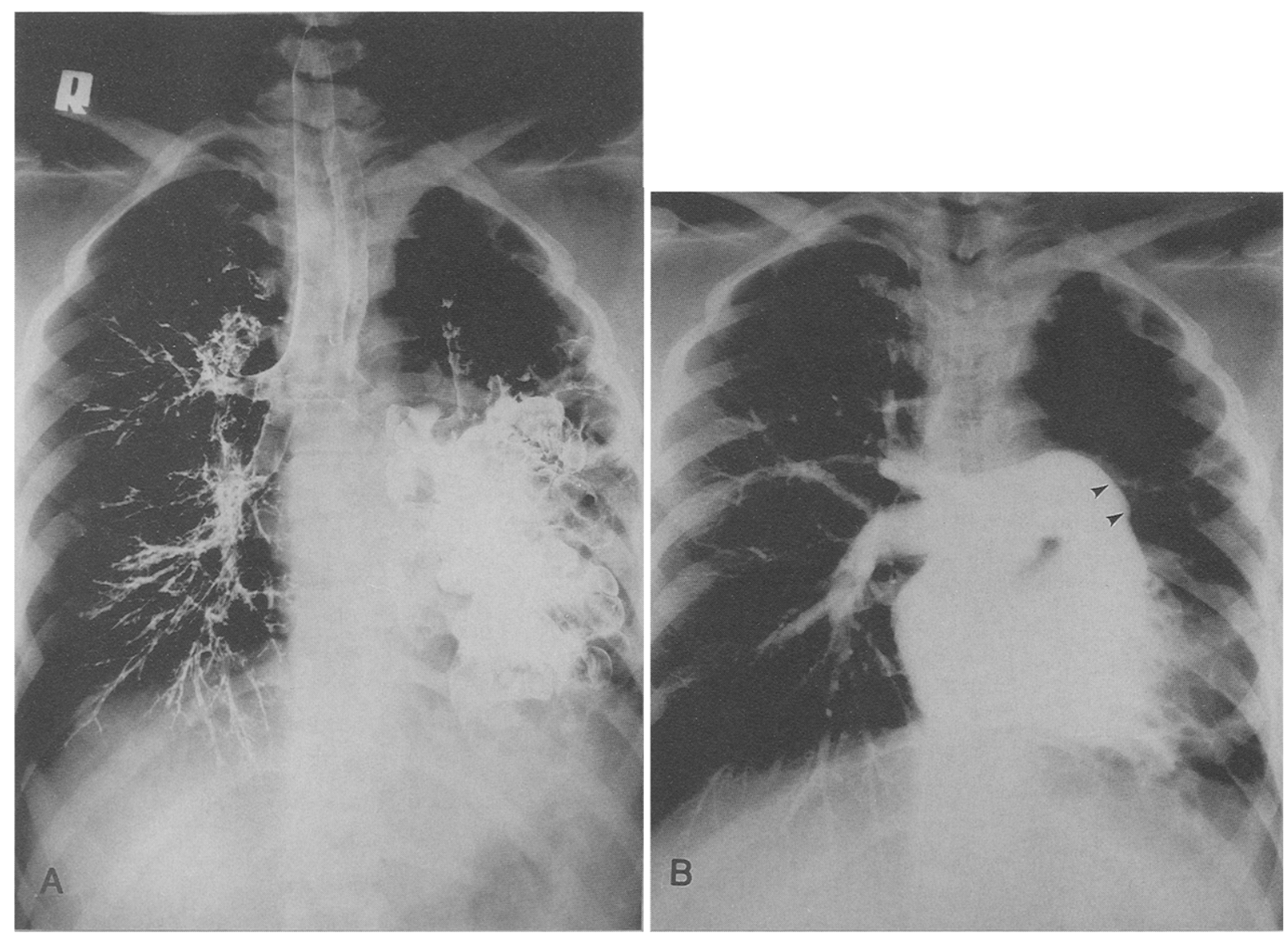

Fig. 6. A, Bronchogram showing cystic bronchiectasis of left lung. B, Pulmonary angiogram of same patient showing false impression of empty left pulmonary artery (small arrows). Injected contrast cannot sufficiently penetrate left pulmonary artery because of hemodynamic block caused by retrograde filling of artery through systemic circulation.

\section{Discussion}

Dilation and hypertrophy of bronchial circulation and extensive bronchopulmonary anastomotic channels in bronchiectasis were described by Leibow, Hales, and Lindskog ${ }^{4}$ in 1949 and Pump ${ }^{5}$ in 1972. Darke and Lewtas ${ }^{6}$ recognized two patterns of bronchopulmonary shunts, those with forward and those with reverse flow. These hemodynamic alterations were generalized descriptive findings and were not linked to a particular type of bronchiectasis.

To my knowledge, hemodynamic alterations specific to each type of bronchiectasis have not been reported before. This study showed that the pattern of pulmonary perfusion in patients with bronchiectasis was not uniform, and hemodynamic alterations specific to each type of bronchiectasis were demonstrated. Thus when the morphologic features of bronchiectasis were correlated with angiographic findings two types of bronchiectasis were recognized, nonperfused and perfused. All lungs with cylindrical bronchiectatic changes were found to be perfused (Fig. 1), whereas those with cystic changes were nonperfused (Fig. 2). This discrepancy in the pattern of pulmonary perfusion reflected the difference in the severity of the disease process. An absent pulmonary artery flow indicated an end-stage disease. This observation was supported by histologic examination of the resected segments. On the other hand, intact pulmonary artery flow indicated a lesser inflammatory process.

In nonperfused bronchiectasis ( $N=19$ lungs), the involved lungs showed absent pulmonary artery flow, retrograde filling of the pulmonary artery through systemic circulation, and cystic bronchiectatic changes. As a result of the pulmonary capillary bed destruction in these lungs, pulmonary capillary 
resistance increased and thus the shunted blood was forced to travel through the pulmonary artery toward the hilum (Fig. 4, B). This phenomenon was described previously as reversal of pulmonary artery flow. ${ }^{6}$ Consequently, the contrast injected during pulmonary angiography cannot sufficiently penetrate into the pulmonary artery because of the hemodynamic block created by the opposing stream of the systemic inflow. Thus a false impression of an empty artery is born (Fig. 6, $A$ and $B$ ). With this understanding in mind, lungs with nonperfused bronchiectasis are unlikely to have a respiratory function with regard to gas exchange. Moreover, this shunt in hemodynamic terms corresponds to a patent ductus arteriosus, an event that might cause left ventricular strain and dyspnea. ${ }^{7}$ Furthermore, the thin-walled dilated bronchial vessels may rupture, thus causing hemoptysis during the course of the disease. ${ }^{8}$ In this series, 9 of the 10 patients who had hemoptysis were known to have nonperfused bronchiectasis, unilaterally in 7 patients and bilaterally in 2 .

On the other hand, in perfused bronchiectasis ( $N=15$ lungs), the involved lungs showed intact pulmonary artery flow and cylindrical bronchiectatic changes (Fig. 1). There was no retrograde filling of the pulmonary artery during thoracic aortography. In these lungs, because the pulmonary capillary bed is relatively intact, the shunted blood will pass from the bronchial circulation through the anastomotic channels to the peripheral pulmonary arteries, finally reaching the pulmonary veins. With this hemodynamic flow in mind, it is possible that lungs in perfused bronchiectasis may continue to have respiratory function with regard to gas exchange.

In view of all these observations, a policy of resecting nonperfused bronchiectasis and preserving the perfused type was adopted. This resection not only served to remove the major source of sepsis with its possible role in spill-over infection to other lung tissues, but also corrected the state of massive bronchopulmonary shunt. As a result, the perfused bronchiectatic parts would be in a better position for the deranged functions to return to normal in time. Seventeen patients were operated on. In the 15 patients with mixed disease, all patients had either excellent $(N$ $=8)$ or $\operatorname{good}(N=7)$ results. All seven patients with good results felt much better and the question of further operation to the other side was not raised. Seven of eight patients with hemoptysis became free of symptoms. On the other hand, two patients with localized bilateral nonperfused disease did not benefit from unilateral resection alone and continued to have hemoptysis. This result suggests that unilateral resection in patients with localized bilateral nonperfused bronchiectasis is inadequate. Instead, staged bilateral resections are likely to offer more favorable outcome.

When dealing with bilateral bronchiectasis, most surgeons operate on the more severely affected side first. ${ }^{9-11}$ Opposite from this approach, Kergin ${ }^{12}$ elected to operate on the less severely affected side first. This sharp swing in the philosophy of surgical management had two pitfalls. First, the term more severely affected side did not involve descriptive criteria to be recognized. Second, operating on the less severely affected side first may result in resection of lung tissues that have a measure of respiratory function.

In the face of the general criticism of the traditional morphologic classification system for bronchiectasis as being inadequate, ${ }^{1,13}$ the proposed functional classification not only reflects the degree of severity of the disease process, but also predicts which type may have a measure of respiratory function. Thus the question of which side to resect and which to preserve is defined more precisely.

\section{REFERENCES}

1. Spencer H. Bronchiectasis. In: Spencer H ed. Pathology of the lung. Vol. 1, 3rd ed. Oxford: Pergamon, 1977:130-49.

2. Roles FC, Tod GS. Bronchiectasis: diagnosis and prognosis in relation to treatment. BMJ 1933;3:639-43.

3. Reid LM. Reduction in bronchial subdivision in bronchiectasis. Thorax 1950;5:233-47.

4. Liebow AA, Hales MR, Lindskog GE. Enlargement of the bronchial arteries and their anastomosis with the pulmonary arteries in bronchiectasis. Am J Pathol 1949;25:211-31.

5. Pump KK. Distribution of bronchial arteries in the human lung. Chest 1972;62:447-51.

6. Darke CS, Lewtas NA. Selective bronchial arteriography in the demonstration of abnormal systemic circulation in the lung. Clin Radiol 1968;19:357-67.

7. Fishman AP. The clinical significance of pulmonary collateral circulation. Circulation 1961;24:677-90.

8. Roosenburg JG, Deenstra H. Bronchial-pulmonary vascular shunts in chronic pulmonary affections. Dis Chest 1954;26: 664-71.

9. Laros CD, van den Bosch JMM, Westermann CJJ, Bergstein PGM, Vanderschueren RGJ, Knaepen PJ. Resection of more than 10 lung segments: a 30 -year survey of 30 bronchiectatic patients. J Thorac Cardiovasc Surg 1988;95:119-23.

10. Borrie J, Lichter I. Surgical treatment of bronchiectasis: ten-year survey. BMJ 1965;2:908-12.

11. Sanderson JM, Kenedy MCS, Johnson MF. Bronchiectasis: results of surgical treatment and conservative management: a review of 393 cases. Thorax 1974;29:407-4.

12. Kergin FG. The surgical treatment of bilateral bronchiectasis. J Thorac Surg 1950;19:257-63.

13. Hood RM. Bacterial diseases of the lung. In: Shields TW, editor. General thoracic surgery. 3rd ed. Philadelphia: Lea and Febiger, 1989:750-71. 